\title{
3D Navigation and Collision Avoidance for a Non-Holonomic Vehicle
}

\author{
Giannis P. Roussos, Dimos V. Dimarogonas, Kostas J. Kyriakopoulos
}

\begin{abstract}
This paper expands the methodology of Navigation Functions for the control of a spherical aircraft-like 3dimensional nonholonomic vehicle. A Dipolar Navigation Function is used to generate a feasible, non-holonomic trajectory for the vehicle that leads from an arbitrary position to the target, in combination with a discontinuous feedback control law that steers the vehicle. The motion model used incorporates the nonholonomic constraints imposed on an aircraft, preventing any movement along the lateral or perpendicular axis, as well as preventing high yaw rotation rates. The control strategy provides guaranteed collision avoidance and convergence, and is supported by non-trivial simulation results.
\end{abstract}

\section{INTRODUCTION}

Potential Field methods in general, and Navigation Functions in particular have been widely used for the control of Nonholonomic robots [4]. Problems addressed include motion planning for a mobile manipulator [18], a mobile robot [19], [15] and multiple manipulators [20]. The aforementioned approaches address 2-dimensional problems, like ground vehicles or aircraft flying on a constant altitude level. There are though applications that are 3-dimensional, like aircraft flying in 3-dimensional space or underwater vehicles, where the above solutions cannot be applied.

Although Navigation Functions as introduced in [16] can be applied to n-dimensional robots and workspaces, holonomic motion model is assumed and required. The adaptation of the methodology to nonholonomic robots is specific for the number of dimensions of each problem, and up to now has only been done for 2-dimensional problems as mentined above, which assume a unicycle-type motion model. The expansion of the existing 2-dimensional approaches to 3dimensional problems is not trivial and requires the assumption of an augmented motion model, which will comply with the kinematic constraints present in the real problem.

Nonholonomic systems require special attention since no time-invariant smooth feedback controller can ba used to stabilize them [6]. Discontinuous control schemes for the stabilization of a single nonholonomic have been proposed by Astolfi [3], Canudas de Wit et. al. [8] and Bloch et. al. [5] Previous work on the control of 3D nonholonomic vehicles include approaches by Aicardi et al. based on a velocity vector field [1], [2] and tracking of a $2 \mathrm{D}$ path that has been expanded empirically to 3D space [12]. It should be noted though that in these approaches no obstacle avoidance

Giannis P. Roussos and Kostas J. Kyriakopoulos are with the Control Systems Lab, Department of Mechanical Engineering, National Technical University of Athens, 9 Heroon Polytechniou Street, Zografou 15780, Greece. email: \{jrous, kkyria\}@mail.ntua.gr

Dimos V. Dimarogonas is with the Automatic Control Lab., School of Electrical Engineering, Royal Institute of Technology, SE-100 44, Stockholm, Sweden. email: dimos@ee.kth.se. method is used, while the bank angle of the vehicle is not controlled.

This paper aims to present a novel method for the control of a fully 3-dimensional vehicle, namely an aircraft that can fly in 3-dimension space. A kinematic controller implementing a fast discontinuous feedback law in real time is used, that provides provable global convergence, along with a Dipolar Navigation Function [18]. The nonholonomic motion model used takes into account the kinematic constraints on the lateral and perpendicular motion that apply on an aircraft. Furthermore, the control law is engineered to keep the yaw rotation rate minimum, as it is common for a conventional fixed-wing aircraft. This control strategy results in the vehicle following a nonholonomic trajectory that avoids collisions with any obstacles or the workspace boundary and leads to the desired configuration. Being a reactive method, this approach is robust with respect to errors in modeling or measurement.

The rest of the paper is organized as follows: section II describes the system and the problem treated, followed by section III, where the Dipolar Navigation Functions framework used in this paper is presented. In section IV, the feedback control scheme used is introduced and analyzed, while section $\mathrm{V}$ includes computer simulation that support the derived results. The conclusions of this paper are summarized in section VI and further research directions are indicated.

\section{SYSTEM AND PROBLEM DEFINITION}

\section{A. A 6-dof, 4-input 3D non-holonomic vehicle}

Consider a 3-dimensional non-holonomic vehicle. The state $\mathbf{n}$ of the vehicle consists of its position $\mathbf{n}_{1}$ and orientation $\mathbf{n}_{2}$ [11]:

$$
\mathbf{n}=\left[\begin{array}{l}
\mathbf{n}_{1} \\
\mathbf{n}_{2}
\end{array}\right], \quad \mathbf{n}_{1}=\left[\begin{array}{l}
x \\
y \\
z
\end{array}\right], \quad \mathbf{n}_{2}=\left[\begin{array}{l}
\phi_{1} \\
\phi_{2} \\
\phi_{3}
\end{array}\right]
$$

where $\left[\begin{array}{lll}\phi_{1} & \phi_{2} & \phi_{3}\end{array}\right]^{T}$ are $x y z$ Euler angles. Let this Earth-fixed coordinate system follow the NED (North-EastDown) convention with $x$ pointing North, $y$ East and $z$ Down. Consequently $\phi_{1}, \phi_{2}, \phi_{3}$ express bank, elevation and azimuth angles respectively.

The motion of the vehicle is described by:

$$
\dot{\mathbf{n}}=\tau=\left[\begin{array}{l}
\tau_{1} \\
\tau_{2}
\end{array}\right]=\left[\begin{array}{l}
\dot{\mathbf{n}}_{1} \\
\dot{\mathbf{n}}_{2}
\end{array}\right]
$$


where $\tau_{1}=\left[\begin{array}{l}f_{1} \\ f_{2} \\ f_{3}\end{array}\right], \quad \tau_{2}=\left[\begin{array}{c}\omega_{1} \\ \omega_{2} \\ \omega_{3}\end{array}\right]$ are the linear and angular velocities respectively.

As stated above the vehicle under consideration is nonholonomic, resembling an aircraft, i.e. there are nonholonomic constraints, which are expressed in the BodyFixed coordinate system which is described below:

Body-Fixed Position and Orientation

$$
\mathbf{r}=\left[\begin{array}{c}
\mathbf{l} \\
\mathbf{a}
\end{array}\right], \quad \mathbf{l}=\left[\begin{array}{l}
l_{1} \\
l_{2} \\
l_{3}
\end{array}\right], \quad \mathbf{a}=\left[\begin{array}{l}
a_{1} \\
a_{2} \\
a_{3}
\end{array}\right]
$$

where $l_{1}$ points forward, $l_{2}$ to the right and $l_{3}$ downwards with respect to the vehicle.

Body-Fixed Linear and Angular Velocities

$$
\mathbf{v}=\left[\begin{array}{c}
\mathbf{v}_{1} \\
\mathbf{v}_{2}
\end{array}\right], \quad \mathbf{v}_{1}=\left[\begin{array}{c}
u \\
v \\
w
\end{array}\right], \quad \mathbf{v}_{2}=\left[\begin{array}{c}
p \\
q \\
r
\end{array}\right]
$$

$p, q, r$ are the body-fixed roll, pitch and yaw rotation rates respectively, as shown in Figure 1.

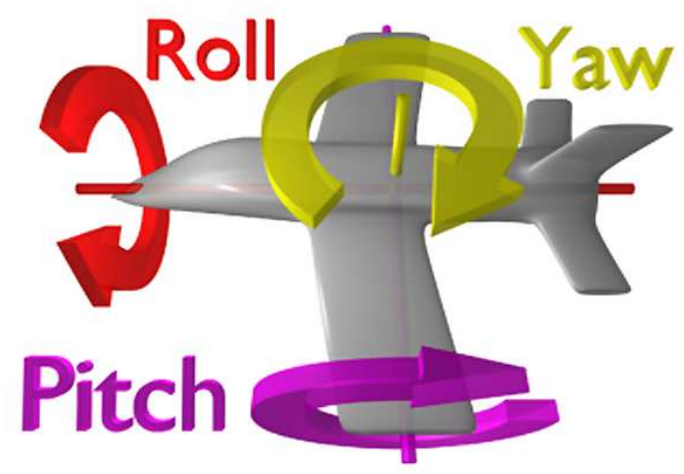

Fig. 1. Body-Fixed rotations

The transformation between body-fixed and earth-fixed velocities is described in [11]:

$$
\begin{aligned}
& \dot{\mathbf{n}}_{1}=\tau_{\mathbf{1}}=\mathbf{J}_{1}\left(\mathbf{n}_{2}\right) \cdot \mathbf{v}_{1} \\
& \dot{\mathbf{n}}_{2}=\tau_{\mathbf{2}}=\mathbf{J}_{2}\left(\mathbf{n}_{2}\right) \cdot \mathbf{v}_{2}
\end{aligned}
$$

where

$$
\begin{aligned}
\mathbf{J}_{1}= & {\left[\begin{array}{cc}
c \phi_{3} c \phi_{2} & -s \phi_{3} c \phi_{1}+c \phi_{3} s \phi_{2} s \phi_{1} \\
s \phi_{3} c \phi_{2} & c \phi_{3} c \phi_{1}+s \phi_{1} s \phi_{2} s \phi_{3} \\
-s \phi_{2} & c \phi_{2} s \phi_{1} \\
s \phi_{3} s \phi_{1}+c \phi_{3} c \phi_{1} s \phi_{2} \\
& -c \phi_{3} s \phi_{3}+s \phi_{2} s \phi_{3} c \phi_{1}
\end{array}\right] } \\
\mathbf{J}_{2}= & {\left[\begin{array}{ccc}
1 & s \phi_{1} t \phi_{2} & c \phi_{1} t \phi_{2} \\
0 & c \phi_{1} & -s \phi_{1} \\
0 & \frac{s \phi_{1}}{c \phi_{2}} & \frac{c \phi_{1}}{c \phi_{2}}
\end{array}\right] }
\end{aligned}
$$

using the notation $s \cdot=\sin (\cdot), c \cdot=\cos (\cdot), t \cdot=\tan (\cdot)$.

The input vector of the non-holonomic system under consideration is

$$
\mathbf{v}_{A}=\left[\begin{array}{llll}
u & \omega_{1} & \omega_{2} & \omega_{3}
\end{array}\right]^{T}
$$

i.e. only the longitudinal (body-fixed) linear velocity $(u)$ and the three rotation rates (earth-fixed) are actuated, while $v=$ $w=0$. Such a model resembles better the motion of an aircraft as it does not allow any movement along the bodyfixed lateral $l_{2}$ or perpendicular $l_{3}$ axis.

Given that according to the selected input vector $v=w=$ 0 , the $2^{\text {nd }}$ and $3^{\text {rd }}$ column of $\mathbf{J}_{1}\left(\mathbf{n}_{2}\right)$ can be omitted to derive the actual kinematic model of the system treated in this paper by combining (1a) and (1b):

$$
\mathbf{n}_{\mathbf{A}}=\tau=\mathbf{R}_{A}\left(\mathbf{n}_{2}\right) \cdot \mathbf{v}_{A}
$$

where $\mathbf{R}_{A}=\left[\begin{array}{cc}\mathbf{J}_{I} & \mathbf{0} \\ \mathbf{0} & \mathbf{J}_{2}\end{array}\right] \in \mathbb{R}^{6 \times 4}, \quad \mathbf{J}_{I}=\left[\begin{array}{c}c \phi_{3} c \phi_{2} \\ s \phi_{3} c \phi_{2} \\ -s \phi_{2}\end{array}\right]$

\section{B. Problem Statement}

The problem under consideration is to design a control law that will steer the vehicle described by 2 to a desired position and orientation, specifically the origin with zero elevation, azimuth and bank angles, i.e. where $x=y=z=\phi_{1}=$ $\phi_{2}=\phi_{3}=0$ while avoiding collision with any obstacles or the boundary $\theta W$ of the given workspace $W \subset R^{3}$. The vehicle as well as the obstacles present in the workspace, and the workspace itself are assumed to be spherical.

\section{Dipolar Navigation Functions}

As discussed above, conventional Navigation Functions are not suitable for the control of a non-holonomic vehicle, as they do not take into account the kinematic constraints that apply on such a vehicle. Use of the original Navigation Function as introduced by Koditschek and Rimon in [16] with a feedback law for the control of a nonholonomic vehicle can lead to undesired behavior, like having the vehicle rotate in place. In order to overcome this difficulty Dipolar Navigation Functions have been developed [20], that offer a significant advantage: the integral lines of the resulting potential field are all tangent to the desired orientation at the origin, eliminating the need for in-place rotation at the origin, as the vehicle is driven there with the desired orientation. This is achieved by using the plane whose normal vector is parallel to the desired orientation, and includes the origin, as an additional artificial obstacle.

The Navigation Function used in this paper is:

$$
V=\frac{\gamma_{d}}{\left(\gamma_{d}^{k}+H_{n h} \cdot G \cdot \beta_{0}\right)^{1 / k}}
$$

where:

$\gamma_{d}=\left\|\mathbf{n}_{1}-\mathbf{n}_{1 d}\right\|^{2}$ is the distance from the destination position $\mathbf{n}_{1 d}$,

$$
G=\prod_{i=1}^{m_{o}} g_{i}
$$




$$
g_{i}=\left\|\mathbf{n}_{1}-\mathbf{n}_{o i}\right\|^{2}-\left(r+r_{i}\right)^{2}, \quad i=1,2, . ., n_{o}
$$

with $r, \mathbf{n}_{o i}, r_{i}$ being the radius of the vehicle, and the position and radius of obstacle $i \in\left[1, \ldots, m_{o}\right]$, respectively, where $m_{0}$ is the number of obstacles. As the workspace is considered spherical with radius $r_{w o r l d}$, the workspace bounding obstacle is $\beta_{0}=r_{\text {world }}^{2}-\left\|\mathbf{n}_{1}\right\|^{2}-r^{2}$.

The factor $H_{n h}$ is what makes the potential field dipolar. As explained before it is responsible for the repulsive potential created by the artificial obstacle used to align the trajectories at the origin with the desired orientation $n_{2 d}$ :

$$
\begin{aligned}
H_{n h} & =\epsilon_{n h}+n_{n h} \\
n_{n h} & =\left\|J_{I d}^{T} \cdot\left(n_{1}-n_{1 d}\right)\right\|^{2} \\
J_{I d} & =J_{I}\left(n_{2 d}\right)
\end{aligned}
$$

where $\epsilon_{n h}$ is a small positive constant. Finally, $k$ is a positive tuning parameter for this class of Navigation Functions.

It was shown in [14] that the potential field created by the Navigation Function defined above has guaranteed navigation properties i.e. it provides global convergence to the destination along with guaranteed collision avoidance. To better demonstrate the properties of a Dipolar Navigation Functions a simple 2-D field is presented in Figure 2, depicting the field in the simple case where no obstacles are present. It can been seen that the surface $x=0$ divides the workspace of radius $r_{w o r l d}=50$ in two parts, and forces all the integral lines to approach the target $(0,0)$ parallel to the $y$ axis.

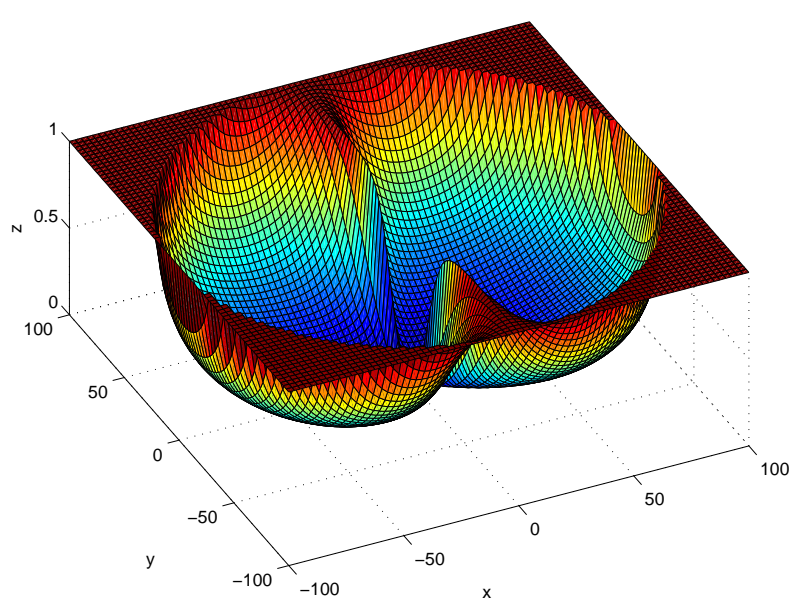

Fig. 2. 2-D Dipolar navigation Function

\section{3D Non-HOLONOMIC NAVIGATION}

\section{A. Control Law}

The proposed kinematic control law is derived from the one proposed in [18], adapted to the 3-dimensional case:

$$
u=-\operatorname{sgn}\left(\mathbf{J}_{I}^{T} \frac{\partial V}{\partial \mathbf{n}_{1}}\right) \cdot \underbrace{\left[k_{u} \cdot\left\|\frac{\partial V}{\partial \mathbf{n}_{1}}\right\|^{2}+k_{z} \cdot\left\|\mathbf{n}_{1}\right\|^{2}\right]}_{\mathbf{F}_{1}\left(\mathbf{n}_{1}\right)} \Rightarrow
$$

$$
\begin{gathered}
u=-\operatorname{sgn}\left(\mathbf{J}_{I}^{T} \frac{\partial V}{\partial \mathbf{n}_{1}}\right) \cdot \mathbf{F}_{1}\left(\mathbf{n}_{1}\right) \\
\omega_{i}=k_{\phi_{i}}\left(\phi_{i d}-\phi_{i}\right), \quad i=1,2,3
\end{gathered}
$$

where $V=V\left(n_{1}\right)$ is the above Dipolar Navigation Function (3), $k_{u}, k_{z}, k_{\phi i}$ are positive real gains and the functions sgn and atan2 are:

$$
\begin{gathered}
\operatorname{sgn}(x) \triangleq \begin{cases}1, & \text { if } x \geq 0 \\
-1, & \text { if } x<0\end{cases} \\
\operatorname{atan} 2(y, x) \triangleq \arg (x, y), \quad(x, y) \in \mathbb{C}
\end{gathered}
$$

The angles $\phi_{i d}$ are defined as follows:

$$
\begin{aligned}
& \phi_{3 d} \triangleq \operatorname{atan} 2\left(\operatorname{sgn}(x) V_{y}, \operatorname{sgn}(x) V_{x}\right) \\
& \phi_{2 d} \triangleq \operatorname{atan} 2\left(-\operatorname{sgn}(x) V_{z}, \sqrt{V_{x}^{2}+V_{y}^{2}}\right) \\
& \phi_{1 d} \triangleq \operatorname{atan} 2\left(\operatorname{sgn}(x) c \phi_{2} \cdot \omega_{3}, \operatorname{sgn}(x) \omega_{2}\right)
\end{aligned}
$$

where $V_{x}=\frac{\partial V}{\partial x}, V_{y}=\frac{\partial V}{\partial y}, V_{z}=\frac{\partial V}{\partial z}$

The control law for the longtitudal velocity drives the vehicle towards either forward or backwards, depending on the sign of the projection of $\frac{\partial V}{\partial \mathbf{n}_{1}}$ on the body-fixed longtitudal $l_{1}$ axis, so that the navigation function is decreasing along the direction of movement.

The control law for elevation and azimuth $\left(\omega_{2}\right.$ and $\omega_{3}$ respectively) is designed so that the vehicle's longtitudal axis steers to align with the Gradient of the Navigation Function. When $x<0$ the vehicle must approach the target moving forward so it steers towards the direction of $-\nabla V$, while when $x>0$ the control law steers the vehicle to the direction of $\nabla V$ in order to approach the target moving backwards.

The bank angle control law $\left(\omega_{1}\right)$ is designed so that the vehicle tends to eliminate the yaw rate $r$ and achieve the required alignment only through pitch rotation $q$, as is preferable for an aircraft. In other words the body-fixed $l_{3}$ axis is driven to align with $\nabla^{2} V$, the curvature vector of the trajectory defined by the Navigation Function. In fact it can be easily shown that the desired bank angle $\phi_{1 d}$ as defined above eliminates the yaw rate in the body-fixed coordinate system:

Using the inverse transformation $\mathbf{v}_{2}=\left[\begin{array}{c}p \\ q \\ r\end{array}\right]=\mathbf{J}_{2}^{-1} \cdot \dot{\mathbf{n}}_{2}$ where $\mathbf{J}_{2}^{-1}=\left[\begin{array}{ccc}1 & 0 & -s \phi_{2} \\ 0 & c \phi_{1} & c \phi_{2} s \phi_{1} \\ 0 & -s \phi_{1} & c \phi_{2} c \phi_{1}\end{array}\right]$ the yaw rate (rotation about the $l_{3}$ axis) can be calculated:

$$
\begin{aligned}
r & =-s \phi_{1} \cdot \omega_{2}+c \phi_{2} c \phi_{1} \cdot \omega_{3} \\
& =-c \phi_{1}\left(t \phi_{1} \cdot \omega_{2}-c \phi_{2} \cdot \omega_{3}\right)
\end{aligned}
$$

assuming that $c \phi_{1 d} \neq 0$. According to the control law $t \phi_{1 d}=$ $\frac{c \phi_{2} \cdot \omega_{3}}{\omega_{2}}$ and consequently $r=0$. In the case where $c \phi_{1 d}=0$ by the definition of $\phi_{1 d}$ we derive that $\omega_{2}=0$ so the yaw rate is: $r=-s \phi_{1} \cdot \omega_{2}+c \phi_{2} c \phi_{1} \cdot \omega_{3}=0$. 


\section{B. Tools from Nonsmooth Analysis}

In order to facilitate the proof that follows, it is useful to review some tool from the analysis of Nonsmooth Systems.

For a differential equation with discontinuous right-hand side we have the following definition:

Definition 1: [10] In the case of a finite dimensional statespace, the vector function $x($.$) is called a Filippov solution$ of $\dot{x}=f(x)$, where $f$ is measurable and essentially locally bounded, if it is absolutely continuous and $\dot{x} \in K[f](x)$ almost everywhere where $K[f](x) \equiv \overline{c o}\left\{\lim _{x_{i} \rightarrow x} f\left(x_{i}\right) \mid x_{i} \notin\right.$ $N_{0}$ \} and $N_{0}$ is a set of measure zero that contains the set of points where $f$ is not differentiable.

Lyapunov theorems have been extended to nonsmooth systems in [17],[7]. The following chain rule provides a calculus for the time derivative of the energy function in the nonsmooth case:

Theorem 1: [17] Let $x$ be a Filippov solution to $\dot{x}=f(x)$ on an interval containing $t$ and $V: \mathbb{R}^{n} \rightarrow \mathbb{R}$ be a Lipschitz and regular function. Then $V(x(t))$ is absolutely continuous, $(d / d t) V(x(t))$ exists almost everywhere and

$$
\frac{d}{d t} V(x(t)) \in^{a . e .} \dot{\widetilde{V}}(x):=\bigcap_{\xi \in \partial V(x(t))} \xi^{T} K[f](x(t))
$$

where "a.e." stands for "almost everywhere".

In this theorem, $\dot{\tilde{V}}$ is Clarke's generalized gradient. The definition of the generalized gradient and of the regularity of a function can be found in [9]. In this paper, the candidate Lyapunov function $V$ we use is smooth and hence regular, while its generalized gradient is a singleton which is equal to its usual gradient everywhere in the state space:

$$
\partial V(x)=\{\nabla V(x)\} \forall x
$$

We use the following nonsmooth version of LaSalle's invariance principle in the sequel:

Theorem 2: [17] Let $\Omega$ be a compact set such that every Filippov solution to the autonomous system $\dot{x}=$ $f(x), x(0)=x\left(t_{0}\right)$ starting in $\Omega$ is unique and remains in $\Omega$ for all $t \geq t_{0}$. Let $V: \Omega \rightarrow R$ be a time independent regular function such that $v \leq 0, \forall v \in \dot{\widetilde{V}}$ (if $\dot{\vec{V}}$ is the empty set then this is trivially satisfied). Define $S=\{x \in \Omega \mid 0 \in \dot{\widetilde{V}}\}$. Then every trajectory in $\Omega$ converges to the largest invariant set, $M$, in the closure of $S$.

Uniqueness of solutions is guaranteed by the above definition of Filippov solutions, along with the measurability assumption of $f([10])$.

\section{Stability Analysis}

Theorem 3: The system (1) under the control law 4 is asymptotically stabilized to $\mathbf{n}=\left[\begin{array}{llllll}0 & 0 & 0 & 0 & 0 & 0\end{array}\right]^{T}$.

Proof: We will use $V$ as a Lyapunov Function Candidate. The generalized time derivative of $V$ is calculated as:

$$
\begin{aligned}
\dot{\tilde{V}} & =\nabla V^{T} \cdot K[\dot{\mathbf{n}}]={\frac{\partial V^{T}}{\partial \mathbf{n}_{1}}}^{T} \cdot K\left[\dot{\mathbf{n}}_{1}\right] \\
& \stackrel{(2)}{=} \frac{\partial V^{T}}{\partial \mathbf{n}_{1}} \cdot \mathbf{J}_{I} K[u]
\end{aligned}
$$

From (4) we derive:

$$
K[u]=K\left[-\operatorname{sgn}\left(\mathbf{J}_{I}^{T} \frac{\partial V}{\partial \mathbf{n}_{1}}\right)\right] \cdot \mathbf{F}_{1}\left(\mathbf{n}_{1}\right)
$$

Finally:

$$
\begin{aligned}
\dot{\tilde{V}} & =\frac{\partial V^{T}}{\partial \mathbf{n}_{1}} \cdot \mathbf{J}_{I} \cdot K\left[-\operatorname{sgn}\left(\mathbf{J}_{I}^{T} \frac{\partial V}{\partial \mathbf{n}_{1}}\right)\right] \cdot \mathbf{F}_{1}\left(\mathbf{n}_{1}\right) \\
& =-\left|\frac{\partial V^{T}}{\partial \mathbf{n}_{1}} \cdot \mathbf{J}_{I}\right| \cdot \mathbf{F}_{1}\left(\mathbf{n}_{1}\right)<=0
\end{aligned}
$$

By the non-smooth version of LaSalle's invariance principle (Theorem 2) we deduce that the system converges to the largest invariant subset included in the set $S \triangleq\{\mathbf{n} \mid 0 \in \dot{\tilde{V}}\}$ Within $S$, we have

$$
\begin{aligned}
0 \in \dot{\tilde{V}} & \Longleftrightarrow \mathbf{J}_{I}^{T} \frac{\partial V}{\partial \mathbf{n}_{1}} \cdot \mathbf{F}_{1}=0 \\
& \Longleftrightarrow\left\{\begin{array}{l}
\mathbf{J}_{I}^{T} \frac{\partial V}{\partial \mathbf{n}_{1}}=0 \\
\text { or } \\
\mathbf{F}_{1}=0
\end{array}\right.
\end{aligned}
$$

The above conditions

$$
\mathbf{F}_{1}=0
$$

and

$$
\mathbf{J}_{I}^{T} \frac{\partial V}{\partial \mathbf{n}_{1}}=0
$$

define two intersecting sets:

$$
S_{1} \triangleq\left\{\mathbf{n} \mid \mathbf{F}_{1}=0\right\} \text { and } S_{2} \triangleq\left\{\mathbf{n} \mid \mathbf{J}_{I}^{T} \frac{\partial V}{\partial \mathbf{n}_{1}}=0\right\}
$$

with $S_{1} \cup S_{2}=S$. (6a) represents the case where $\left\|\frac{\partial V}{\partial \mathbf{n}_{1}}\right\|=$ 0 i.e. the gradient of the potential field is zero, and the current position is the origin i.e. $\mathrm{x}=\mathrm{y}=\mathrm{z}=0$, while the second condition (6b) is satisfied when the gradient vector is normal to the aircraft's longtitudal axis.

For $S_{1}$ where $\mathbf{F}_{1}=0$, by the definition of $\mathbf{F}_{1}$ we have $\frac{\partial V}{\partial \mathbf{n}_{i}}=0$ and consequently $\frac{\partial V}{\partial l_{i}}=0, \quad i=1,2,3$, which yields

$$
\phi_{i d}=0, \quad i=1,2,3
$$

Let us then define the subset

$$
S_{3} \triangleq S_{1} \bigcap\left\{\mathbf{n} \mid \phi_{i}=0, \quad i=1,2,3\right\} \subset S_{1}
$$

which is the origin with the desired zero azimuth, elevation and bank angles. By the control law (4) then we deduce:

$$
\omega_{i}=0 \stackrel{(4 b),(7)}{\Longleftrightarrow} \phi_{i}=0 \quad \text { for } i=1,2,3
$$

Furthermore $u=0$ inside $S_{1} \supset S_{3}$, so $S_{3}$ is the only invariant subset of $S_{1}$.

For the set $S \backslash S_{1} \subset S_{2}$, where $\mathbf{J}_{I}^{T} \frac{\partial V}{\partial \mathbf{n}_{1}}=\frac{\partial V}{\partial l_{1}}=0$ and $\mathbf{F}_{1} \neq 0$, the potential field's gradient is non-zero and normal to the aircraft's longtitudal axis. In this case, as it will be 
proven by contradiction below, at least one of the elevation and azimuth angular velocities $\left(\omega_{2}, \omega_{3}\right)$ is non-zero and steers the vehicle away from this set:

Suppose that the set $S \backslash S_{1}$ is invariant, then $\omega_{i}=0$ for $i=1,2,3$, which by (4b) means that

$$
\phi_{i d}=\phi_{i}
$$

Then by the definition of $\phi_{2 d}$ and $\phi_{3 d}$ we derive:

$$
\begin{array}{ll}
s \phi_{2}=\frac{\operatorname{sgn}(x) V_{z}}{\sqrt{V_{x}^{2}+V_{y}^{2}+V_{z}^{2}}} \quad s \phi_{3}=\frac{\operatorname{sgn}(x) V_{y}}{\sqrt{V_{x}^{2}+V_{y}^{2}}} \\
c \phi_{2}=\frac{\sqrt{V_{x}^{2}+V_{y}^{2}}}{\sqrt{V_{x}^{2}+V_{y}^{2}+V_{z}^{2}}} \quad c \phi_{3}=\frac{\operatorname{sgn}(x) V_{x}}{\sqrt{V_{x}^{2}+V_{y}^{2}}}
\end{array}
$$

when $\sqrt{V_{x}^{2}+V_{y}^{2}} \neq 0$ so that $s \phi_{3}, c \phi_{3}$ can be calculated as above.

Since the Navigation Function $\mathrm{V}$ is polar with exactly one minimum of zero value at the origin, $\mathbf{F}_{1} \neq 0$ means that $\left\|\frac{\partial V}{\partial \mathbf{n}_{1}}\right\|^{2} \neq 0$ and $\left\|\mathbf{n}_{1}\right\|^{2} \neq 0$, i.e. outside $S_{1}$ the navigation function and its gradient $\|\nabla V\|=\sqrt{V_{x}^{2}+V_{y}^{2}+V_{z}^{2}}$ are always non-zero and consequently $s \phi_{2}, c \phi_{2}$ can be calculated in the above way. Substituting in (6b) we get: $\operatorname{sgn}(x)\left[V_{x}{ }^{2}+V_{y}{ }^{2}+V_{z}{ }^{2}\right]=0$ which is not possible outside $S_{1}$. Thus it has been shown that when $\sqrt{V_{x}^{2}+V_{y}^{2}} \neq 0$ inside the set $S \backslash S_{1}$ the condition (8) cannot hold and consequently by (4b) at least one of $\omega_{2}, \omega_{3}$ is non-zero.

In the trivial case where inside $S \backslash S_{1} \sqrt{V_{x}^{2}+V_{y}^{2}}=0 \Leftrightarrow$ $V_{x}=V_{y}=0$ (i.e. the gradient is perpendicular) then $V_{z} \neq 0$ and $s \phi_{2} \neq 0$, so (6b) yields $\operatorname{sgn}(x) s \phi_{2} V_{z}=0$ which cannot hold, proving again that (8) cannot hold in this case either.

We have showed then that in the set $S \backslash S_{1}$ at least one of the angular velocities $\omega_{2}, \omega_{3}$ is non-zero, so the set is not invariant. This proves that the only invariant set in $S$, where every trajectory of the system converges under the proposed control law, is $S_{3}$, i.e. the origin with zero elevation, azimouth and bank angle.

\section{Simulation}

The control strategy presented above has been used on a computer simulation. The test case consisted of a workspace with $r_{\text {world }}=150$, containing 3 obstacles of various radii scattered in the workspace. The initial configuration of the vehicle has been set at

$$
\mathbf{n}_{\text {init }}=\left[\begin{array}{llllll}
-90 & 90 & 30 & 0 & \frac{\pi}{4} & -\frac{3 \pi}{2}
\end{array}\right]^{T}
$$

The goal is to drive the vehicle to the origin with zero azimuth, elevation and bank angles, where

$$
x=y=z=\phi_{1}=\phi_{2}=\phi_{3}=0
$$

The results are presented in the following Figures, showing the trajectory of the vehicle from 3 different viewing angles.

As Figures 3, 4 and 5 demonstrate, the vehicle follows a feasible, nonholonomic 3-dimensional path avoiding all

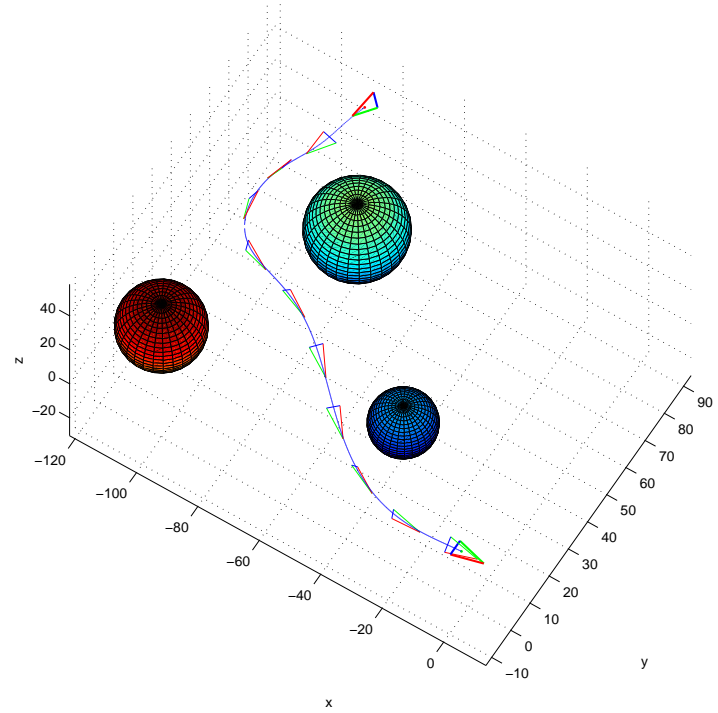

Fig. 3.

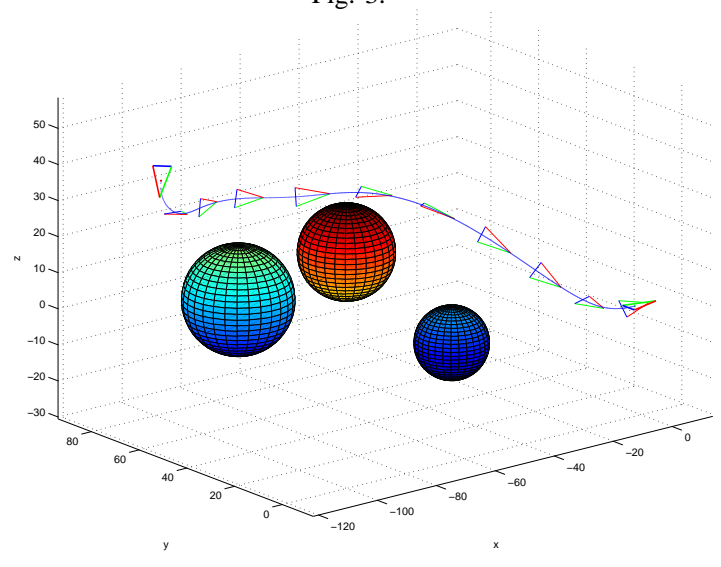

Fig. 4.

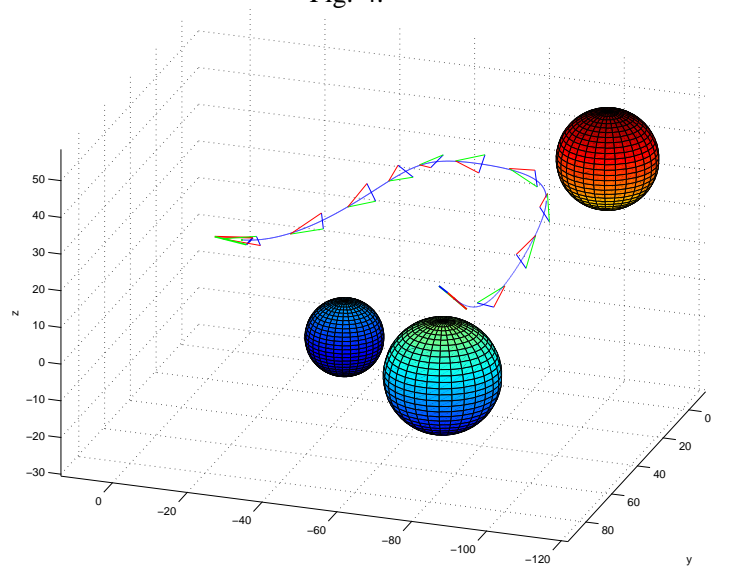

Fig. 5.

the obstacles, and converges to the origin with the desired orientation. Furthermore, it can be seen that the bank angle control law rotates the vehicle so that the body-fixed yaw rotation rate is maintained low, as intended. The efficiency of this is further demonstrated in Figure 6, where the yaw 


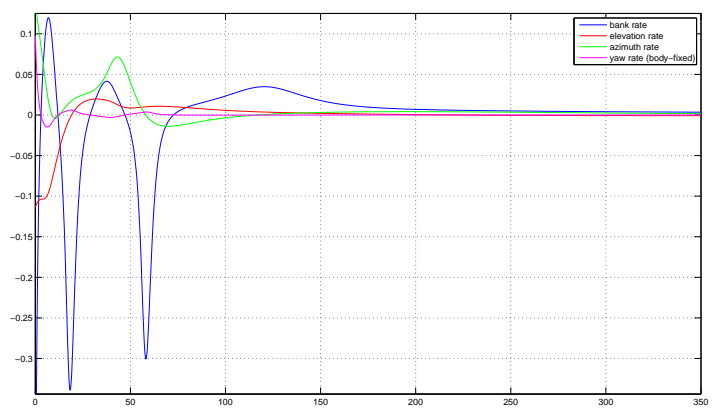

Fig. 6. Rotation rates history

rotation rate is presented, in comparison with the 3 earthfixed rotation rates.

\section{CONCLUSIONS}

This paper presents a novel application of the Navigation Functions' methodology to the control of a 3-dimensional aircraft-like non-holonomic vehicle. The control scheme uses a Navigation Functions along with a discontinuous feedback control law. The result is a 3-dimensional non-holonomic trajectory leading to the target position with the desired orientation.The use of a feedback law makes the control strategy robust with respect to measurement and modeling errors, while the Navigation Function provides guaranteed global convergence and collision avoidance.

Further research includes the use of body-fixed velocities for the control, so that more non-holonomic constraints can be imposed (like zero yaw rotation rate). Furthermore, tuning the Navigation Function in order to impose a certain curvature vector at the origin can offer better control over the bank angle. Finally the expansion of the methodology for multiple vehicles is expected.

\section{ACKNOWLEDGEMENTS}

The first and third author of this paper want to acknowledge the contribution of the European Commission through contract iFLY ("Safety, Complexity and Responsibility based design and validation of highly automated Air Traffic Management"), No TREN/07/FP6AE/S07.71574/037180.

The second author's work was done within the TAISAURES project which is supported by the Swedish Governmental Agency for Innovation Systems (VINNOVA) and Swedish Defence Materiel Administration (FMV). He was also supported by the KTH ACCESS Linnaeus Center, the Swedish Research Council, the Swedish Foundation for Strategic Research, and the EU NoE HYCON.

\section{REFERENCES}

[1] M. Aicardi, G. Cannata, G. Casalino, and G. Indiveri. Guidance of $3 \mathrm{~d}$ underwater non-holonomic vehicle via projection on holonomic solutions. Symposium on Underwater Robotic Technology SURT 2000, World Automation, 2000.

[2] M. Aicardi, G. Casalino, and G. Indiveri. Closed loop control of 3d underactuated vehicles via velocity field tracking. 2001 IEEE/ASME International Conference on Advanced Intelligent Mechatronics, 2001.

[3] Alessandro Astolfi. Exponential stabilization of a wheeled mobile robot via discontinuous control. ASME Journal of Dynamic Systems Measurement and Control, 121:121-125, 1999.
[4] Anthony M. Bloch. Nonholonomic Mechanics and Control. SpringerVerlag NY, 2003.

[5] Anthony M. Bloch, Sergey V. Drakunov, and Michael K. Kinyon. Stabilization of nonholonomic systems using isospectral flows. SIAM Journal on Control and Optimization, 38(3):855-874, 2000.

[6] Roger W. Brockett. Asymptotic stability and feedback stabilization. pages 181-191, 1983.

[7] F. Ceragioli. Discontinuous ordinary differential equations and stabilization. PhD thesis, Dept. of Mathematics, Universita di Firenze, 2000

[8] Carlos Canudas de Wit and O. J. Sordalen. Exponential stabilization of mobile robots with nonholonomics constraints. IEEE Transaction on Automatic Control, 37(11), 1992.

[9] F.Clarke. Optimization and Nonsmooth Analysis. Addison-Wesley, 1983.

[10] A. Filippov. Differential equations with discontinuous right-hand sides. Kluwer Academic Publishers, 1998.

[11] Thor I. Fossen. Guidance and Control of Ocean Vehicles. 1994.

[12] G.Ambrosino, M.Ariola, U.Ciniglio, F.Corraro, A.Pironti, and M.Virgilio. Algorithms for $3 \mathrm{~d}$ uav path generation and tracking. Proceedings of the 45th IEEE Conference on Decision \& Control, 2006.

[13] Savvas G. Loizou, Dimos V. Dimarogonas, and Kostas J. Kyriakopoulos. Decentralized feedback stabilization of multiple nonholonomic agents. Proceedings of the 2004 International Conference on Robotics and Automation, pages 3012-3017, 2004.

[14] Savvas G. Loizou and Kostas J. Kyriakopoulos. Closed loop navigation for multiple non-holonomic vehicles. Control Systems Lab - NTUA, Technical Report No. 2.

[15] Gabriel A. D. Lopes and Daniel E. Koditschek. Navigation functions for dynamical, nonholonomically constrained mechanical systems. pages 135-155, 2006.

[16] Elor Rimon and Daniel E. Koditschek. Exact robot navigation using artificial potential functions. IEEE Transactions on Robotics and Automation, pages 501-508, 1992.

[17] Daniel Shevitz and Brad Paden. Lyapunov stability theory of nonsmooth systems. IEEE Transactions on Automatic Control, 39(9):19101914, 1994.

[18] Herbert G. Tanner and Kostas J. Kyriakopoulos. Nonholonomic motion planning for mobile manipulators. Proceedings of the 2000 IEEE International Conference on Robotics \& Automation, pages 12331238,2000

[19] Herbert G. Tanner, Savvas Loizou, and Kostas J. Kyriakopoulos. Nonholonomic stabilization with collision avoidance for mobile robots. Proceedings of the 2001 IEEE/RSJ International Conference on Intelligent Robots and Systems, pages 1220-1225, 2001.

[20] Herbert G. Tanner, Savvas Loizou, and Kostas J. Kyriakopoulos. Nonholonomic navigation and control of cooperating mobile manipulators. IEEE Transactions on Robotics and Automation, pages 53-64, 2003. 